\title{
ANTHOCYANIN-RICH PHENOLIC EXTRACTS OF BLACK CHOKEBERRY (ARONIA MELANOCARPA) ATTENUATE INFLAMMATION INDUCED BY LIPOPOLYSACCHARIDE IN RAW 264.7 CELLS
}

\author{
MA, Y. $.^{1 \#}-\mathrm{WEI}, \mathrm{L}^{2 \#}-\mathrm{XU}, \mathrm{Q} .{ }^{1}-\mathrm{WANG}, \mathrm{Y}^{2}-\mathrm{LI}, \mathrm{Z}^{1}{ }^{1}-\mathrm{ZHOU}, \mathrm{W} .{ }^{3}-\mathrm{MENG}, \mathrm{X} .{ }^{4 *}$ \\ ${ }^{1}$ Center of Experiment Teaching, Shenyang Normal University, Shenyang 110034, China \\ (e-mail/phone:ma1976@126.com/+86-138-0401-0160-Y.Ma; 1183759107@qq.com-Q. \\ Xu; Lizheholle@163.com-Z.Li) \\ ${ }^{2}$ College of Food Science, Shenyang Agriculture University, Shenyang 110866, China \\ (e-mail/phone: weilulusy@126.com/+86-158-2745-6126-L.Wei; 308579439@qq.com-Y. \\ Wang) \\ ${ }^{3}$ Food Inspect Monitoring, Experiment Center, Ctr Chaoyang, Chaoyang 122000, China \\ (e-mail:3518299636@qq.com)
}

${ }^{4}$ Shenyang Agricultural University, Shenyang, Liaoning 110866, China

*Corresponding author

e-mail: mengxjsy@126.com, phone: +86-133-9011-7107

${ }^{\#}$ These authors have contributed equally to this work

(Received $5^{\text {th }}$ Sep 2020; accepted $21^{\text {st }}$ Dec 2020)

\begin{abstract}
Aronia melanocarpa berries and their extracts, have become well known for their notable health benefits. The present study investigated the anti-inflammatory effect of anthocyanin-rich phenolic extracts of Ame (Aronia melanocarpa) in LPS (lipopolysaccharide)-stimulated RAW 264.7 murine macrophage cells. The results showed that Ame pre-treatment significantly ameliorated oxidative stress and inflammatory biomarker activities, as evidenced by reductions in the production of ROS (reactive oxygen specie), MDA (malondialdehyde), and NO (nitric oxide), as well as suppression of iNOS (inducible nitric oxide synthase), COX-2 (cyclooxygenase) and PGE2 (prostaglandin E2) mRNA levels; remarkably elevated the level of anti-inflammatory cytokine IL (interleukin)-10; and reduced the levels of the pro-inflammatory cytokines IL-1p, IL-6, and TNF-a (tumour necrosis factor). Additionally, we observed an attenuation of the cell apoptosis levels and the mRNA expression of apoptosis factors such as caspase-3 and caspase-9. In summary, the results highlight the health benefit of Ame against inflammation in LPS-stimulated RAW 264.7 cells.
\end{abstract}

Keywords: Aronia melanocarpa anthocyanins, anti-inflammatory activity, cell apoptosis, apoptosis factors

\section{Introduction}

Our inclusion of berries in the diet is gaining popularity due to their richness in healthbeneficial nutrients, such as phenolic compounds, flavonoids, anthocyanidins and antioxidant vitamins (Hwang et al., 2014b). For the past several years, a growing amount of evidence has indicated that the consumption of plant foods rich in polyphenolic compounds is correlated with a lower risk of the development for oxidative stress-related diseases and has a beneficial effect beyond the actions of vitamins (Denev et al., 2012).

Recent studies have shown an increasing interest in Aronia melanocarpa (black chokeberry), which belongs to the Rosaceae family and originates from North America (Jakobek et al., 2012). It is a rich source of phenolic compounds, particularly 
proanthocyanidins and anthocyanins. Many earlier studies have shown that the concentrations phenolic compounds is many times higher than those in apples, red raspberries, blackberries, sweet rowanberries, blackthorn, sweet cherry, sour cherry, blueberries, raspberries, etc. (Castañeda-Ovando et al., 2009; Pellati et al., 2004; Polat et al., 2017). Aronia berries are extensively used for the production of juices, preserves, jams, wines and food colorants (Simić et al., 2016), and a number of health benefits have been ascribed to their intake (Mcdougall et al., 2016). In vitro and in vivo studies have demonstrated that black chokeberry has a wide range of positive effects, such as inhibition of cancer cell proliferation (Tao et al., 2017), antimutagenic effects, neuroprotective effects (Lee et al., 2017) and antidiabetic capabilities (Ciocoiu et al., 2017). It also displays several health-promoting properties in relation to chronic diseases, especially gastroprotective, hepatoprotective, and cardioprotective effects, which are related to its ant-inflammatory properties (Jurikova et al., 2017). Thus, aronia berries have potential as functional food ingredients. Inflammation is a complex physiopathological phenomenon that is mediated by activated inflammatory cells of the immune system, including macrophages (Yoon et al., 2012). It may induce various chronic diseases including cancer, cardiovascular diseases, Alzheimer's disease, type II diabetes, arthritis, metabolic syndrome, neurological diseases, and infectious diseases (Ahn et al., 2015; Hwang et al., 2014a). Lipopolysaccharide (LPS) is an endotoxin that is a potent inducer of inflammation and triggers the activation of macrophages that later release biomarkers of oxidative stress and inflammatory mediators, which then induce apoptosis (Khan et al., 2016).

Oxidative stress is considered a harmful disequilibrium between the generation and removal of radicals including lipid peroxidation products and ROS (reactive oxygen species) (Sivasinprasasn et al., 2016). Furthermore, ROS may damage biological molecules such as lipids, proteins and DNA and are crucial promoters of inflammation and cardiovascular disease (Rop et al., 2010). Inflammatory mediators include cell cytokines. Stimulated macrophages will release large amounts of cell cytokines such as IL (interleukin)-Ip, IL-6, IL-10, TNF-a (tumour necrosis factor) and other inflammatory mediators such as PGE2 (prostaglandin E2), iNOS (inducible nitric oxide synthase) and COX-2 (cyclooxygenase) during the inflammation process (Zdařilová et al., 2010). Thus, inhibition of the production of these inflammatory mediators is an important target in the treatment of inflammatory diseases. It has been reported that oxidative stress can impair function and trigger apoptosis (Isaak et al., 2017). Caspase-3 and Caspase-9 are directly involved in the process of apoptosis and are important pro-apoptotic molecules.

Compared with the synthetic anti-inflammation constituents, natural bioactive ingredients have higher efficiency and are economical. Besides, synthetic anti-inflammation constituents may exhibit toxicity and side-effects. Thus, it is necessary to search for more natural bioactive resources. Natural plant extracts including Aronia melanocarpa extract have shown beneficial effects on inflammation via the reduction of damage due to oxidative stress, apoptosis and modulation of inflammation cytokine expression.

Recently, works by Ah Ra Goh have indicated that Aronia melanocarpa extract exerts anti-inflammatory activities by inhibiting expression of pro-inflammatory mediators and ROS generation in HaCaT cells (Goh et al., 2016). Similar results were also obtained showing that intake of anthocyanin-rich black chokeberry juice can inhibit both the release of TNF-a, IL-6 and IL-8 in human peripheral monocytes and the activation of the NF-KB pathway in RAW 264.7 macrophage cells (Appel et al., 2015).

Herein, we investigated the anti-inflammatory effects and action mechanisms of Aronia melanocarpa extract and how it exerts an anti-inflammatory effect in LPS 
(lipopolysaccharide)-inflamed murine RAW264.7 macrophage cells in the present study. To the best of our knowledge, this is the first study that evaluated the antiinflammatory properties of Aronia melanocarpa extract from three aspects: oxidative stress, inflammatory mediators, and apoptosis. The study further investigated whether Aronia melanocarpa extract could be used as a potential novel ingredient in antiinflammatory health products or as a candidate drug for the prevention of inflammation.

\section{Materials and methods}

\section{Materials and reagents}

Ripe fruits of Aronia melanocarpa cultivars ("Fukangyuan Number 1") were

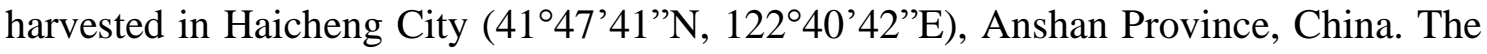
Aronia melanocarpa were submitted to $60 \%$ alcoholic extraction, and the extracts were preserved at $-20{ }^{\circ} \mathrm{C}$ for use. All the chemicals and reagents were purchased from Wanlei and Dingguo Biological Technology Co., Ltd. (Shenyang, Liaoning, China).

\section{Phytochemical analysis}

\section{Anthocyanin phenolic acids}

Anthocyanins were quantified by HPLC (High Performance Liquid Chromatography) experiments according to a previously described method (Wang et al., 2016). The column was a Dikma Platisil C18 column $(4.6 \mathrm{~mm} \times 250 \mathrm{~mm}$ inner diameter, $5 \mathrm{pm})$, and the solvent system used was $0.5 \%$ water solution in formic acid (A) and $100 \%$ HPLC grade acetonitrile (B) (elution conditions: 0-40 min from 0 to $40 \% \mathrm{~B}$; 40-45 min, $40-45 \% \mathrm{~B}, 45-52 \mathrm{~min}, 0 \% \mathrm{~B}$; flow rate $0.7 \mathrm{~mL} \mathrm{~min}^{-1}$, injection volumes were $20 \mu \mathrm{L}$ ). Data were recorded at $520 \mathrm{~nm}$. Anthocyanin components were quantified based on the calibration curves of structurally related external standards (cyanidin-3-glucoside). The standard concentration ranged from $0.5 \times 10^{-3}$ to $1.5 \times 10^{-3} \mathrm{mg} / \mathrm{mL}$.

The phenolic acids were quantified using an HPLC system (Agilent 1100, Palo Alto, CA, USA) at $210 \mathrm{~nm}$. They were separated using a $0.1 \%$ water solution of formic acid as solvent $\mathrm{A}$ and HPLC grade acetonitrile in $0.1 \%$ formic acid as solvent B (elution conditions: $0-45 \min$ from 0 to $45 \% \mathrm{~B}$; $45-52 \min 0 \% \mathrm{~B}$; flowrate $=0.7 \mathrm{ml} \mathrm{min}^{-1}$; injection volumes $10 \mu 1$ ). Each component was quantified based on the calibration curves of the structurally related external standards (gallic acid, protocatechuic acid, phydroxybenzoic acid, chlorogenic acid, caffeic acid, benzoic acid, p-coumaric acid, ferulic acid, cinnamic acid). The standard concentration ranged from $2.5 \times 10^{-5}$ to $50 \times 10^{-5} \mathrm{mg} / \mathrm{mL}$ (Polat et al., 2017).

\section{Total polyphenol content, anthocyanins, flavonoids and proanthocyanidin}

The slightly modified method of Wang et al. (2016a) was applied to evaluate the total polyphenol content in the Aronia melanocarpa extracts. In short, $0.5 \mathrm{~mL}$ of the sample and $3 \mathrm{~mL}$ of Folin-Ciocalteu's reagent were incubated in the dark for $5 \mathrm{~min}$ at room temperature. Then, $2.4 \mathrm{~mL}$ of $7.5 \%$ sodium carbonate was injected and incubated for $2 \mathrm{~h}$ at room temperature in the dark. The absorbance values of the reaction mixture were then measured at $765 \mathrm{~nm}$. Gallic acid $(0-100 \mu \mathrm{g} / \mathrm{mL})$ was used as the standard (mg GAE/g).

The total anthocyanin content in the samples was determined by the $\mathrm{pH}$-differential method. Briefly, 0.025 M potassium chloride and 0.4 M sodium acetate were separately 
adjusted to $\mathrm{pH} 1.0$ and $\mathrm{pH} 4.5$ with hydrochloric acid. Next, $1 \mathrm{~mL}$ of the sample and $24 \mathrm{~mL}$ of buffer were incubated in the dark at room temperature for $15 \mathrm{~min}$, and the absorbance was measured at $510 \mathrm{~nm}$ and $700 \mathrm{~nm}$ with distilled water as the blank control. Finally, the total anthocyanin content was calculated according to the formula given by Wang et al. (2016a).

The total flavonoid content of chokeberry cultivars was evaluated by the $\mathrm{NaNO}_{2}$ AlNOs-NaOH method. The sample solution $(0.1 \mathrm{ml})$ was mixed with $4 \mathrm{ml}$ of ethanol $(30 \%)$, followed by $0.5 \mathrm{~mL}$ of $\mathrm{NaNO}_{2}(10 \%), 0.5 \mathrm{~mL}$ of $\mathrm{Al}(\mathrm{NO} 3)_{3}(10 \%)$ and $4 \mathrm{ml}$ of $\mathrm{NaOH}(4 \%)$. After incubation at room temperature for $30 \mathrm{~min}$, the absorbance was measured at $510 \mathrm{~nm}$ and the total flavonoids content was calculated as rutin equivalents (mg RE/g).

The proanthocyanidin content of black chokeberries was evaluated by the method of Pedro et al. (2015) with modifications. Briefly, the sample solutions ( $1 \mathrm{~mL})$ were mixed with $5 \mathrm{ml}$ of $1 \%$ vanillin (1.0 g vanillin in $100 \mathrm{~mL}$ of methanol) and $10 \%$ conc- $\mathrm{H}_{2} \mathrm{SO}_{4}$ $\left(10 \mathrm{~mL}\right.$ conc- $\mathrm{H}_{2} \mathrm{SO}_{4}$ in $100 \mathrm{~mL}$ of methanol) at a proportion of $1: 1(\mathrm{v} / \mathrm{v})$. After incubation for $30 \mathrm{~min}$ at $25{ }^{\circ} \mathrm{C}$, the absorbance was measured at $500 \mathrm{~nm}$ and the proanthocyanidin content was calculated as catechin (mg CE/g).

\section{Measurement of anti-inflammatory capacity}

\section{Cell culture and treatment}

The mouse macrophage cell line Raw 264.7 (obtained from the cell bank of the Chinese Academy of Sciences, Shanghai, China) was cultured in complete DMEM (Dulbecco's Modified Eagle's Medium) with 10\% heat-inactivated fetal bovine serum. Briefly, Raw 264.7 cells were seeded in 96-well and 6-well plates at a density of $10^{3}$ cells/well and incubated at $37{ }^{\circ} \mathrm{C}, 5 \% \mathrm{CO}_{2}$ in a humidified incubator and allowed to attach overnight before the experiments. The Ame (Aronia melanocarpa) was filtered through a microfiltration membrane $(0.22 \mu \mathrm{m})$ prior to addition to the culture media and was resuspended in DMEM to achieve a final concentration of $50 \mu \mathrm{g} / \mathrm{mL}$. Cells were treated with the following:
i. DMEM only (control group)
ii. Ame added for $4 \mathrm{~h}$ daily for two consecutive days (Ame group)
iii. $\quad$ LPS at $1 \mu \mathrm{g} / \mathrm{mL}$ for $24 \mathrm{~h}$ (LPS group)
iv. Ame for $4 \mathrm{~h}$ daily for two consecutive days and then LPS at $1 \mu \mathrm{g} / \mathrm{mL}$ for another $24 \mathrm{~h}$ (LPS/Ame group)

The combination of dose/time for the Ame and LPS treatments was established based on preliminary MTT viability assays (data not shown). The cells and cell supernatants were collected and immediately frozen $\left(-20^{\circ} \mathrm{C}\right.$ or $\left.-80^{\circ} \mathrm{C}\right)$ until analysis.

\section{Determination of ROS level}

The measurement of ROS was performed according to the instructions given by the manufacturer of the kit (Shenyang Wanlei Bioengineering Institute, Shenyang, China). Briefly, DCFH-DA (2',7'-Dichlorodihydrofluorescein diacetate) was added to the serum-free culture medium to a final concentration of $10 \mu \mathrm{M}$. After incubation for $20 \mathrm{~min}$ at $37^{\circ} \mathrm{C}$, the cells were then collected and centrifuged at $1000 \mathrm{~g}$ for $10 \mathrm{~min}$. Supernatants were removed carefully, after which the cells were resuspended in $200 \mu \mathrm{L}$ of PBS and the fluorescence easily measured at $\lambda_{\text {exication }} 490 \mathrm{~nm}$ and $\lambda_{\text {emission }} 530 \mathrm{~nm}$. ROS production levels for each treatment were normalized to the non-stimulated control and expressed as \% control. 


\section{Determination of lipid peroxidation}

Lipid peroxidation was determined by measuring the MDA (malondialdehyde) in cells using a commercial MDA Kit (Shenyang Wanlei Bioengineering Institute, Shenyang, China). The absorbance was read on a microplate reader at $532 \mathrm{~nm}$. MDA production levels for each treatment were normalized to the non-stimulated control and expressed as \% control.

\section{Nitric oxide (NO) inhibitory activity}

The measurement of NO (nitric oxide) in Raw 264.7 cells was performed using a commercial NO kit (Nanjing Jiancheng Bioengineering Institute, Nanjing, China) according to the manufacturer's instructions. The optical density was measured using a microplate reader at $550 \mathrm{~nm}$. NO production levels for each treatment were normalized to the non-stimulated control and expressed as percent control.

\section{RNA isolation and reverse transcription-polymerase chain reaction (RT-PCR)}

Total RNA was isolated using a total RNA extraction kit, and 1 pg of RNA was used for cDNA synthesis using a Super M-MLV reverse transcriptase kit (BioTeke Corporation, Beijing, China) according to the manufacturer's protocol. After amplifying cDNA using real-time quantitative PCR with a SYBR green PCR Master Mix (Solarbio Life Sciences Institute, Beijing, China) according to our protocol, the levels of mRNA expression were quantified using an RT-PCR (RNA isolation and reverse transcription-polymerase chain reaction) system (ExicyclerTM 96, Bioneer, Daejeon, Korea). The primers' details used in this study are presented in Table 1 . The thermal cycling parameters were as follows: 1 cycle at $94{ }^{\circ} \mathrm{C}$ for $5 \mathrm{~min}, 94^{\circ} \mathrm{C}$ for $10 \mathrm{~s}, 60^{\circ} \mathrm{C}$ for $20 \mathrm{~s}$ and $72^{\circ} \mathrm{C}$ for $30 \mathrm{~s}$, followed by 40 cycles of $2.5 \mathrm{~min}$ at $7{ }^{\circ} \mathrm{C}, 1.5 \mathrm{~min}$ at $40{ }^{\circ} \mathrm{C}$, melting for $34 \mathrm{~s}$ at $60^{\circ} \mathrm{C}$ to $94{ }^{\circ} \mathrm{C}$ and $2 \mathrm{~min}$ at $25{ }^{\circ} \mathrm{C}$. The nucleotide sequence of each primer and the size of the PCR products are shown in Table 1. mRNA expression was analyzed using the $2^{-\Delta \Delta \mathrm{C}_{\mathrm{r}}}$ method and normalized with respect to the expression of the $\mathrm{p}$-actin housekeeping gene.

\section{Apoptosis assay by flow cytometry}

Annexin V-FITC/PI staining was performed to measure apoptosis by using an Apoptosis Detection Kit (Kaiji Bioengineering Institute, Nanjing, China) according to the manufacturer's protocol. Briefly, cells from different treatment groups were collected and washed twice with cold PBS (phosphate buffered saline). The cell supernatant was carefully removed by centrifugation at $300 \times \mathrm{g}$ for $5 \mathrm{~min}$, and the cells were resuspended in $500 \mu \mathrm{L}$ binding buffer, followed by the addition of $5 \mu \mathrm{L}$ of Annexin V-FITC and $5 \mu \mathrm{L}$ of PI (propidium iodide). After 15 min of incubation at room temperature in darkness, each sample was analyzed with flow cytometry (C 6, Becton Dickinson and Company, New Jersey, USA).

\section{Statistical analysis}

All experimental results are expressed as means and were performed in triplicate; the data in the tables and figures represent mean values \pm standard deviation. One-way ANOVA (Analysis of variance) with Duncan's multiple range test was used to examine the differences between groups. Significant differences were considered to be $p<0.05$ or $p<0.01$. 
Table 1. The oligonucleotide primer sets for the real-time PCR analysis

\begin{tabular}{c|c|c|c|c}
\hline Name & Sequence (5'-3') & Length & Tm & size \\
\hline PGE2 F & GCCATTATGACCATCACCTTCG & 22 & 61.8 & 250 \\
PGE2 R & GCCATTATGACCATCACCTTCG & 23 & 60.1 & \\
iNOS F & GCAGGGAATCTTGGAGCGAGTTG & 23 & 67.1 & 139 \\
iNOS R & GTAGGTGAGGGCTTGGCTGAGTG & 23 & 65 & \\
COX-2 F & TTCCTCCCGTAGCAGATGACT & 21 & 58.9 & 205 \\
COX-2 R & AACCCAGGTCCTCGCTTA & 18 & 55.1 & \\
IL- $\beta$ F & TGGTACATCAGCACCTCACA & 20 & 54.7 & 132 \\
IL- $\beta$ R & GAAGGCATTAGAAACAGTCC & 20 & 51.5 & \\
IL-6 F & TGTATGAACAACGATGATGCAC & 22 & 56.7 & 194 \\
IL-6 R & CTGGCTTTGTCTTTCTTGTT & 20 & 52.2 & \\
IL-10 F & GAAGACAATAACTGCACCCACT & 22 & 56.2 & 162 \\
IL-10 R & ACCCAAGTAACCCTTAAAGTCC & 22 & 56.5 & \\
caspase-3 F & TGACTGGAAAGCCGAAAC & 18 & 53.7 & 203 \\
caspase-3 R & GGACTGGATGAACCACGAC & 19 & 55 & \\
caspase-9 F & CACTGCCTCATCATCAACAA & 20 & 54.5 & 168 \\
caspase-9 R & CATCAAAGCCGTGACCAT & 18 & 54.1 & \\
TNF-a F & AGAAAGCATGATCCGCGAC & 19 & 58.3 & 236 \\
TNF-a R & TTGTGAGTGTGAGGGTCTGG & 20 & 55.8 & \\
P-actin F & CTGTGCCCATCTACGAGGGCTAT & 23 & 64.5 & 155 \\
P-actin R & TTTGATGTCACGCACGATTTCC & 22 & 63.2 & \\
\hline
\end{tabular}

\section{Results and discussion}

\section{Chemical composition}

The detailed composition and contents of the Aronia melanocarpa extract are shown in Table 2. In the present study, 4 individual anthocyanins and 9 phenolic acids were identified in Ame. Ame possessed a high content of phenolic contents $(655.11 \pm 21.6 \mathrm{mg}$ gallic acid equivalent/g), anthocyanins (195.76 $\pm 19.43 \mathrm{mg} / \mathrm{g})$ and flavonoids $(75.28 \pm 6.82 \mathrm{mg}$ rutin equivalent/g). HPLC-MS/MS (Liquid chromatography-mass spectrometry/mass spectrometry) analysis detected 4 anthocyanin pigments, with cyanidin 3-galactoside $(92.44 \pm 8.96 \mathrm{mg} / \mathrm{g})$ and cyanidin 3 -arabinoside $(27.7 \pm 4.51 \mathrm{mg} / \mathrm{g})$ being the most representative anthocyanins in Ame. The present results indicated that anthocyanins predominated in the phenolic fractions of Ame, which are responsible for several beneficial actions in human health. That consistent with previous reports (Parzonko et al., 2015). The 9 main phenolic acids identified using HPLC methodology allowed the identification of Ame polyphenols based on standards. The most common and abundant phenolic acid compounds identified in Ame are benzoic acid and chlorogenic acid. These results are consistent with those previously reports (Polat et al., 2017).

\section{The effects of Ame on biomarkers of oxidative stress: ROS and MDA}

A sustained pro-inflammatory state, characterized by excessive ROS production, is the common denominator in the development, progression, and complication of many diseases (Gasparrini et al., 2017). For this reason, the measurement of ROS intracellular production could represent a very useful parameter to quantify oxidative stress induced by LPS. To investigate whether treatment with Ame influences LPS-induced ROS production, ROS was measured. According to our results, Ame itself caused no increase 
in basal ROS generation in RAW 264.7 macrophages $(p>0.05)$, while Ame significantly $(0.01<p<0.05)$ suppressed the intracellular ROS production of LPSstimulated RAW 264.7 macrophages based on $36 \%$ or higher changes relative to the LPS-stimulated controls (Fig. 1A). It suggesting that Ame phytochemicals may play a role in health maintenance by reducing oxidative stress (Goh et al., 2016).

Table 2. Quantification and determination of total and individual phenolic compounds in Ame

\begin{tabular}{c|c|c|c}
\hline Compound $^{\mathbf{a}}$ & Ame $\mathbf{( m g / g )}$ & ${\text { Phenolic } \text { acid }^{\mathbf{c}}}^{\mathbf{g}}$ & Ame $\mathbf{( m g / g )}$ \\
\hline Total phenolic content $^{\prime}$ & $655.11 \pm 21.6$ & Protocatechuic acid & $0.102 \pm 0.07$ \\
Flavonoid content & $75.28 \pm 6.82$ & P-hydroxybenzoic acid & $0.055 \pm 0.02$ \\
Proanthocyanidin content & $0.06 \pm 0.02$ & Chlorogenic acid & $1.643 \pm 0.31$ \\
Anthocyanins & & Caffeic acid & $0.686 \pm 0.17$ \\
Cyanidin 3-galactoside & $92.44 \pm 8.96$ & Benzoic acid & $10.206 \pm 1.22$ \\
Cyanidin 3-glucoside & $4.04 \pm 0.37$ & P-coumaric acid & $0.295 \pm 0.08$ \\
Cyanidin 3-arabinoside & $27.7 \pm 4.51$ & Ferulic acid & $0.267 \pm 0.10$ \\
Cyanidin 3-xyloside & $6.21 \pm 0.54$ & Cinnamic acid & $0.253 \pm 0.15$ \\
Total anthocyanins & $195.76 \pm 19.43$ & Gallic acid & $0.045 \pm 0.02$ \\
\hline
\end{tabular}

Values are means $\pm \mathrm{SD}(\mathrm{n}=3)$

andividual phenolic compounds were compared with standard reference compounds

${ }^{b}$ Identified using HPLC-ESI-MS ${ }_{2}$

'Identified using HPLC

Lipid peroxidation is a free-radical-mediated chain reaction involving several types of free radicals, which could be arrested through enzymatic means or by free radical scavenging by antioxidants and is considered one of the major manifestations of oxidative stress (Divya et al., 2015). Therefore, we used a lipid peroxidation assay to strengthen our findings in the above-mentioned regions. In the present study, we assessed the effect of topical administration of Ame during mouse macrophage cell LPS exposure by measuring the concentration of the short-chain aldehyde, MDA, which is the by-product of lipid peroxidation. As shown in Figure 1B, LPS application obviously enhanced MAD (machine analysis display). Pre-treatment with Ame significantly $(0.01<p<0.05)$ reduced the MDA level, suggesting that Ame might be involved in the prevention of inflammation dysfunction via reducing the oxidative stress level in macrophages. These results obtained for the first time with Aronia melanocarpa, which are consistent with those previously reported by several authors, who tested the efficacy of different bioactive compounds against LPS-induced damage in macrophage cell models (Bak et al., 2013; Gasparrini et al., 2017; Lee et al., 2013).

\section{The effects of Ame on inflammatory mediators}

\section{Inflammatory mediators: NO, PGE2, iNOS and COX-2}

NO, PGE2, iNOS and COX-2 are the most important indicators for assessing inflammation injury. iNOS expression can increase the production of NO (Lee et al., 2013), which is also reported to affect the activity of COX-2 (Li and Wang, 2011). Similar to iNOS, COX-2 is also an inducible pro-inflammatory enzyme. COX-2 can convert arachidonic acid into PGE2, which can contribute to the pain and swelling associated with inflammation (Lee et al., 2013). 

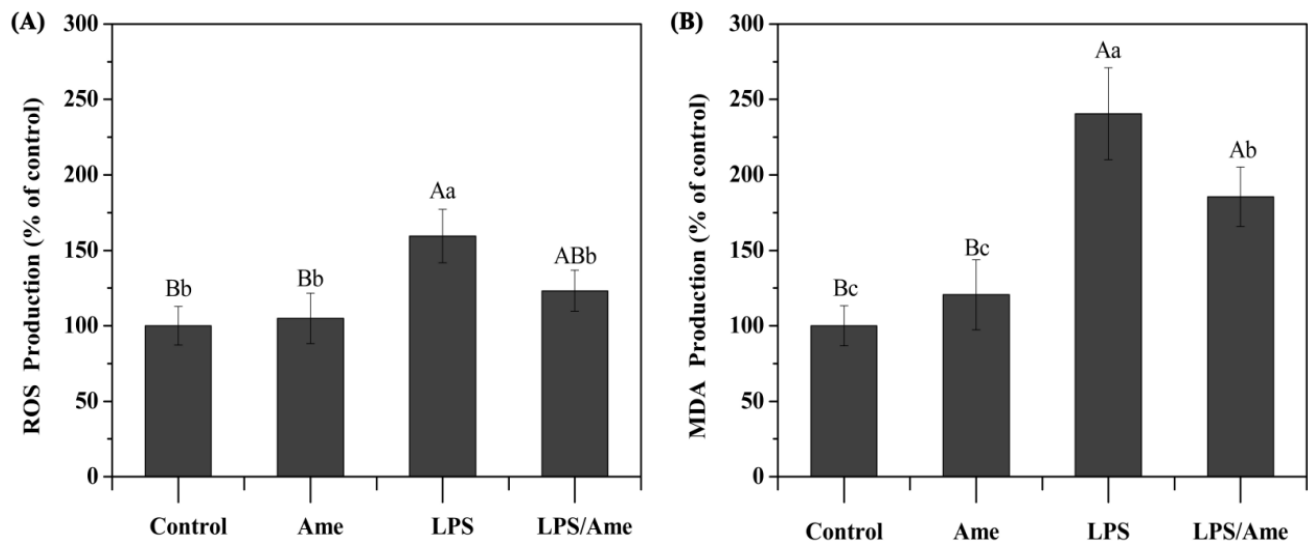

Figure 1. The effects of Ame on biomarkers of oxidative stress (A and B): ROS and MDA. Ame, treatment with Ame alone; LPS, treatment with LPS alone; LPS/Ame, Ame pre-treatment followed by LPS treatment. The results are presented as the mean $\pm S D(n=3)$. One-way (ANOVA) followed by Duncan's multiple range tests was performed to analyze the statistical differences among means. The different superscript lowercase letters denote significant differences between groups, and $p<0.05$ or $p<0.01$ was considered statistically significant

The effects of Ame on the level of NO and PGE2 in the culture media of RAW 264.7 cells were determined after $24 \mathrm{~h}$ treatment with $1 \mu \mathrm{g} / \mathrm{mL}$ LPS. As shown in Figure $2 A$, there was no basal NO production during the incubation with only Ame without LPS $(p>0.05)$. After treatment with LPS, the NO concentration in the medium increased by approximately 2.45 -fold (245.29\%) compared to the control (100\%). However, Ame at $50 \mu \mathrm{g} / \mathrm{mL}$ significantly inhibited the production of NO $(p<0.01)$.

The inhibitory effects of Ame on LPS-induced PGE2 secretion in RAW 264.7 cells were determined using RT-PCR (real-time polymerase chain reaction). As shown in Figure 2B, when the macrophages were not stimulated with LPS, PGE2 was almost undetectable in the medium with or without Ame $(p>0.05)$, while treatment with LPS caused an elevated production of PGE2 $(p<0.01)$, which was greatly reduced by Ame $(p<0.01)$. In this study, Ame effectively decreased NO production and PGE2 mRNA expression, indicating that Ame might be useful for suppressing the inflammatory process.

To investigate whether the Ame fractions had inhibitory activities against NO and PGE2 production via inhibition of iNOS and COX-2, RT-PCR analysis was used. As shown in Figure $2 C$ and $D$, the expression of iNOS and COX-2 mRNA only showed an almost undetectable change between the unstimulated group and the Ame pre-treatment group. After LPS treatment, iNOS and COX-2 mRNA expression were markedly increased, whereas cotreatment with Ame significantly suppressed the expression of iNOS and COX-2 mRNA. These results are consistent with the inhibitory effect of Ame on NO and PGE2 release. The inhibitory profiles of Ame for iNOS and COX-2 overlapped with the profiles for NO and PGE2 production. On the basis of these results, it was concluded that Ame inhibited iNOS-mediated NO and COX-2-mediated PGE2 production. A similar finding was reported by a previous study, where blueberry extract was found to alleviate NO, PGE2 and COX-2 (Xu et al., 2016).

\section{Inflammatory cytokines: $I L-1 \beta, I L-6, T N F-a$ and IL-10}

Cytokines are a critical component of immune defense, but, on the other hand, inappropriate or excessive production of IL-1 $\beta$, IL-6, TNF-a and IL-10 has been linked 
with the pathogenesis of a number of chronic inflammatory diseases (Yaqoob et al., 2010). IL-1 $\beta$ is known to induce fever and inflammation, finally leading to apoptosis. Moreover, it has been shown that IL-6 can be secreted by macrophages in response to specific microbial molecules to initiate the innate immune system (Yoon et al., 2009) and is a crucial checkpoint regulator of neutrophil trafficking by orchestrating chemokine production and leukocyte apoptosis (Fang et al., 2015). TNF-a is involved in many different cellular processes, including the production of numerous cytokines and acute phase proteins, and thus contributes to many pathophysiologic processes (Liu, 2005). On the other hand, IL-10 is a type of anti-inflammatory factor that down-regulates inflammatory responses and plays a role in inflammatory mediators of antagonism.


Figure 2. The effects of Ame on inflammatory mediators (A, B, C and D): NO, PGE2, iNOS and COX-2. Ame, treatment with Ame alone; LPS, treatment with LPS alone; LPS/Ame, Ame pretreatment followed by LPS treatment. The results are presented as the mean $\pm S D(n=3)$. Oneway (ANOVA) followed by Duncan's multiple range test was performed to analyze the statistical differences among means. The different superscript lowercase letters denote significant differences between groups, and $p<0.05$ or $p<0.01$ was considered statistically significant

As shown in Figure $3 A, B$ and $C$, Ame itself caused no increase in the aforementioned cytokine mRNA expression in RAW 264.7 macrophages $(p>0.05)$. When compared with cells treated with LPS alone, Ame resulted in an approximately $66 \%, 16 \%$ and $21 \%$ reduction in the mRNA expression of IL-1p, IL-6 and TNF-a, respectively. All of these reductions were significant $(p<0.05)$, especially IL-1 $\beta$ $(p<0.01)$. A different trend was found for IL-10: in unstimulated cells, IL-10 secretion increased to $104 \%(p>0.05)$ of the control with $50 \mu \mathrm{g} / \mathrm{mL}$ Ame; in this case, an 
increase in IL-10 mRNA expression was observed with Ame + LPS treatments, with a significant increase $(p<0.01)$ compared to LPS-treated cells. The present results demonstrated that bioactive compounds in response to Ame exhibited a significant adjustment of inflammatory cytokines in RAW264.7 macrophages after exposure to LPS that helps decrease inflammatory damage.
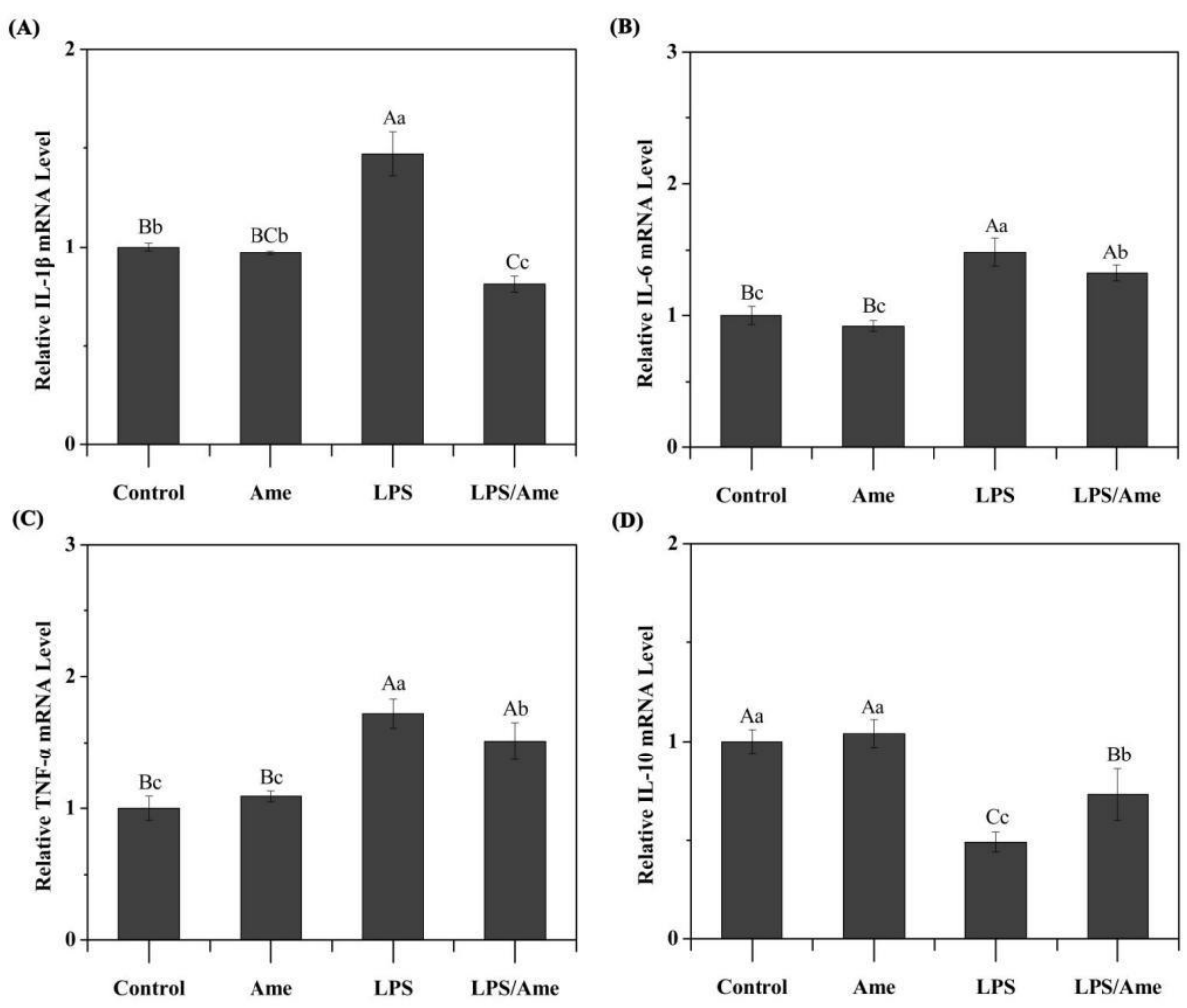

Figure 3. The effects of Ame on inflammatory cytokines (A, B, C and D): IL-1p, IL-6, TNF-a and IL-10. Ame, treatment with Ame alone; LPS, treatment with LPS alone; LPS/Ame, Ame pretreatment followed by LPS treatment. The results are presented as the mean $\pm S D(n=3)$. Oneway (ANOVA) followed by Duncan's multiple range tests was performed to analyze the statistical

differences among means. The different superscript lowercase letters denote significant differences between groups, and $p<0.05$ or $p<0.01$ was considered statistically significant

Previous reports (Appel et al., 2015) have suggested that the phenolics from chokeberry concentrate inhibit the release of TNF-a, IL-6 and IL-8 in LPS-induced RAW264.7 macrophages. These results are in line with the data obtained in other studies performed on RAW264.7 macrophages in which the expression of pro- and antiinflammatory cytokines induced by LPS was improved by Ame (Appel et al., 2015) and other different bioactive compounds from strawberries (Gasparrini et al., 2017), blueberries (Wang et al., 2017), and Lonicera caerulea L (Wang et al., 2016b).

\section{The effects of Ame on apoptosis}

Biomarkers of apoptosis: caspase-3 and caspase-9

Caspases are known as important mediators of apoptosis and contribute to leading cells undergoing apoptosis to irreversible cell death. Caspase- 3 and caspase-9 play major roles 
in the pathway of extrinsic apoptosis and endogenous apoptosis (Meng et al., 2017). Studies have shown that upregulation of caspase and TNF-a is an important factor in apoptosis (Chu et al., 2016). Therefore, we measured the activation of caspase- 3 and caspase-9 with RT-PCR. As shown in Figure $4 A$ and $B$, Ame itself caused no increase in caspase-3 and caspase-9 mRNA expression in RAW 264.7 macrophages $(p>0.05)$, while treatment with LPS for $24 \mathrm{~h}$ resulted in the activation of caspase- 3 and caspase- 9 as is apparent in comparison to the control groups $(p<0.01)$, but Ame treatment at $50 \mu \mathrm{g} / \mathrm{mL}$ significantly protected against caspase-3 and caspase-9 activation $(p<0.01)$. Interestingly, the mRNA expression of caspase-3 and caspase-9 after Ame + LPS treatment was similar to the control group $(p>0.05)$. These observations indicate that Ame can down-regulate mRNA expression of caspase-3 and caspase-9, thereby reducing macrophage cell death, which is an important step in preserving the immune system following inflammatory damage. In contrast to previous studies, we first evaluated the anti-inflammatory activity of Ame by exploring one aspect of apoptosis.
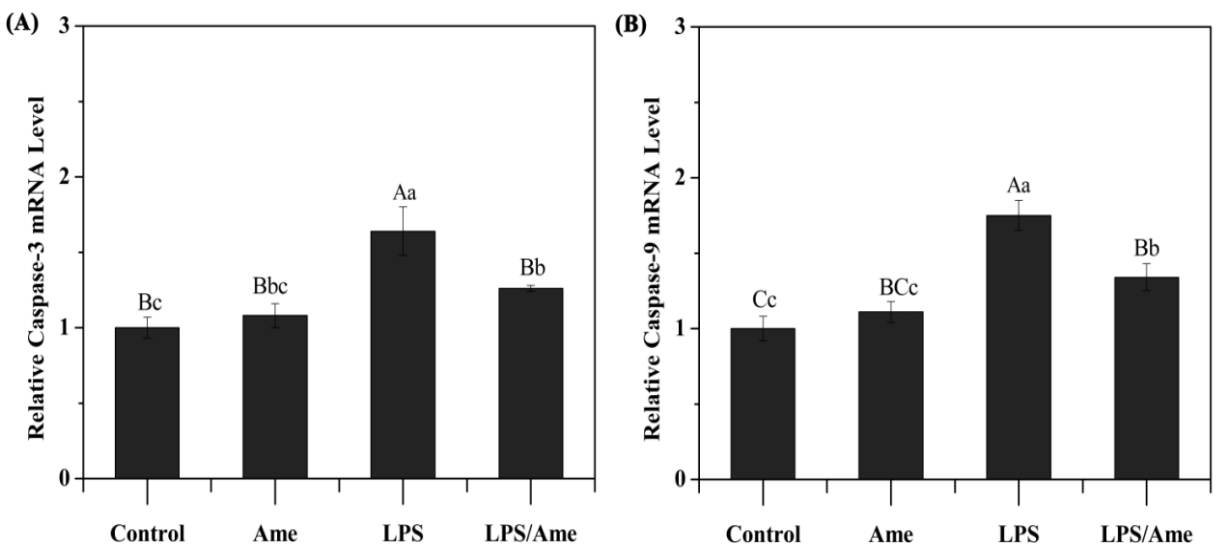

Figure 4. The effects of Ame on biomarkers of apoptosis (A and B): caspase-3 and caspase-9. Ame, treatment with Ame alone; LPS, treatment with LPS alone; LPS/Ame, Ame pre-treatment followed by LPS treatment. The results are presented as the mean $\pm S D(n=3)$. One-way (ANOVA) followed by Duncan's multiple range test was performed to analyze the statistical differences among means. The different superscript lowercase letters denote significant differences between groups, and $p<0.05$ or $p<0.01$ was considered statistically significant

\section{Apoptosis detected by flow cytometry}

Flow cytometry was used to determine in which phase of the cell cycle the RAW 264.7 macrophages had accumulated and whether apoptotic events occurred in response to LPS exposure. As shown in Figure 5, in RAW 264.7 macrophages cells, LPS exposure groups resulted in typical apoptotic changes in the cells compared with cells from the untreated groups. When treated with $50 \mathrm{~g} / \mathrm{mL}$ Ame, the apoptotic rate remarkably reduced the severity of apoptosis compared with the LPS groups (early apoptosis: $12.23 \%$ vs. $11.05 \%$, late apoptosis: $21.65 \%$ vs. $11.01 \%$, total apoptosis: $33.88 \%$ vs. $23.06 \%$, respectively, $p<0.05$ ). Treatment with Ame could therefore block RAW 264.7 cell apoptosis. Furthermore, Ame itself caused no increase in the apoptosis rate in RAW 264.7 macrophages $(p>0.05)$. The results showed that the early stages of apoptosis were lower than the late apoptosis. This also suggests that the reduction in cell inflammatory damage from the Aronia melanocarpa extracts was at least partially due to apoptosis of the RAW 264.7 cells. 
(A) $r$
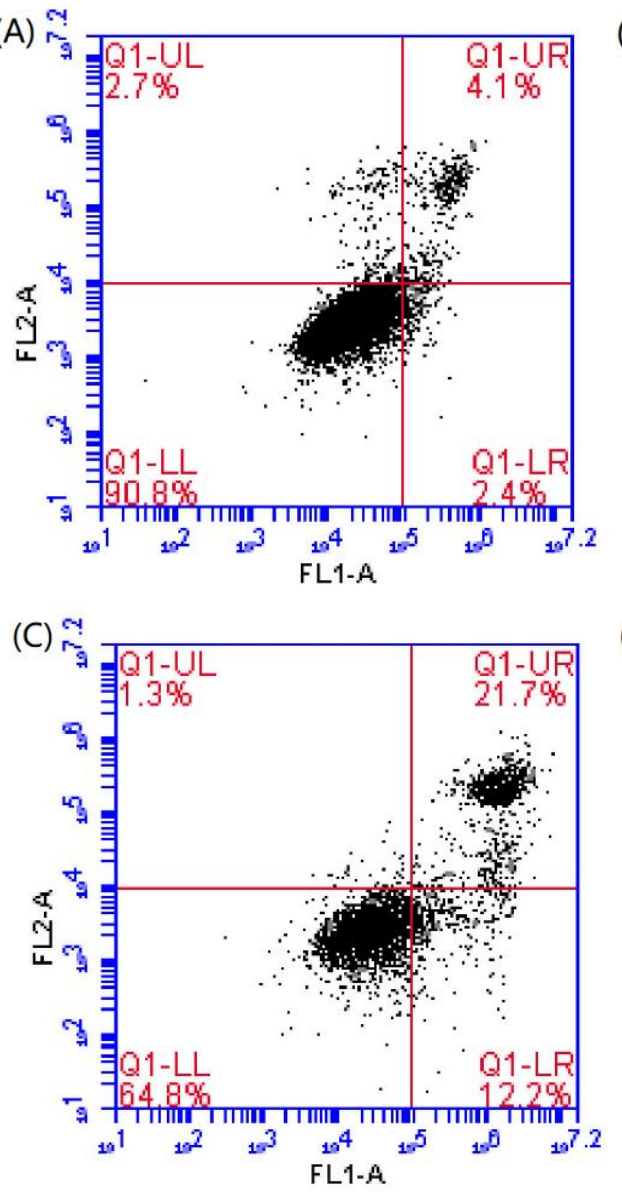

(B)

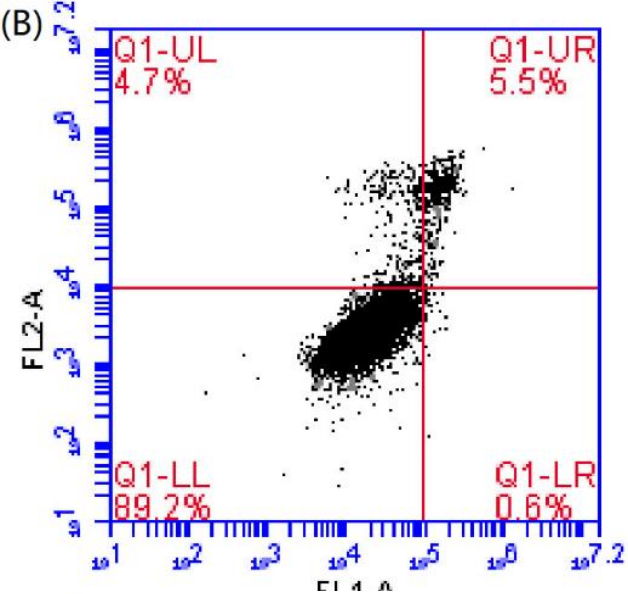

(D)

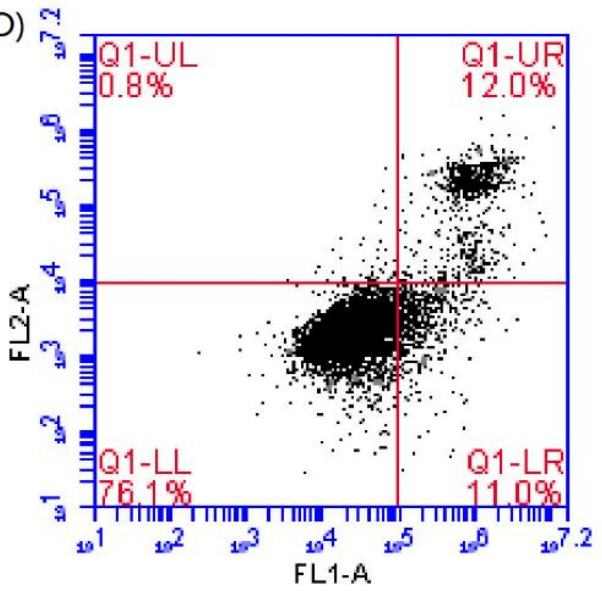

(E)

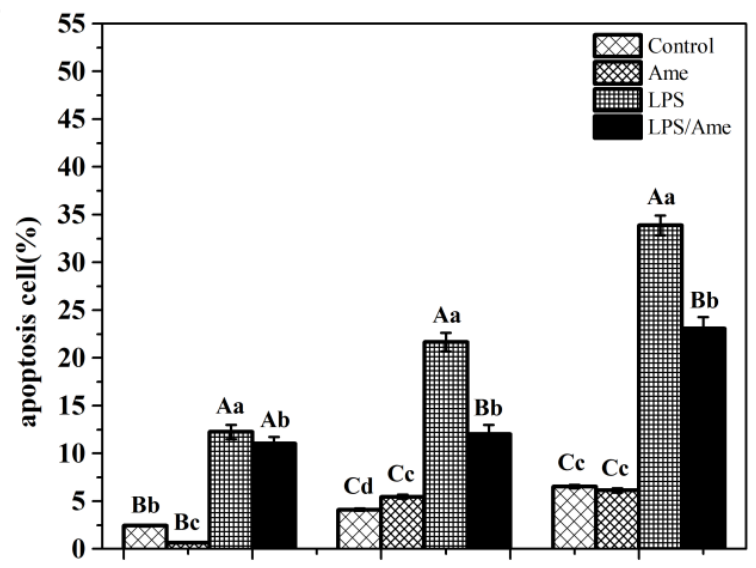

the early apoptosis the late apoptosis the total apoptosis

Figure 5. The effects of Ame on apoptosis detected by flow cytometry. Images of flow cytometry detection of RAW 264.7 macrophage apoptosis (A, B, C and D). (A) Control; (B) Ame, treatment with Ame alone; (C) LPS, treatment with LPS alone; (D) LPS/Ame, Ame pretreatment followed by LPS treatment. UL, necrotic cells. UR, late stage apoptotic cells. LR, early stage apoptotic cells. LL, live cells. (E) The results of flow cytometry detection of RAW 264.7 macrophage apoptosis in the early stage and late stage of apoptosis. The results are presented as the mean $\pm S D(n=3)$. One-way (ANOVA) followed by Duncan's multiple range test was performed to analyze the statistical differences among means. The different superscript lowercase letters denote significant differences between groups, and $p<0.05$ or $p<0.01$ was considered statistically significant 


\section{Conclusions}

In summary, we found that the Aronia melanocarpa extract, containing anthocyanins as its main phenolic components, could reduce LPS-induced inflammation by inhibiting the development of oxidative stress (via evaluation of ROS and MDA), regulating the activity of inflammatory mediators (NO, PGE2, iNOS and COX-2), attenuating the production of pro-inflammatory cytokines (IL-1 $\beta$ IL-6 and TNF-a), and increasing the expression of anti-inflammatory cytokines (IL-10). Aronia melanocarpa extract also attenuated the LPS-induced biomarkers of apoptosis: caspase- 3 and caspase-9 prevented apoptosis of RAW 264.7 macrophages. Our results demonstrate that polyphenolic substances in Aronia melanocarpa extracts, especially anthocyanins, possess antiinflammatory activities, Aronia melanocarpa extract has the potential to be developed as a novel ingredient in anti-inflammatory health products or as a candidate drug for the prevention of inflammation.

In this paper, Aronia melanocarpa were extracted and enriched to obtain freeze-dried powder with high anthocyanin content. The components in freeze-dried powder were identified by various methods, and its antioxidant ability was measured in vitro. Then its anti-inflammatory properties were explored from oxidative stress, inflammatory factors and apoptosis. On the basis of this paper, we can further supplement the paper and study the anthocyanin extract of Liriodendron nigra in more details and more perfectly.

The mechanism of anthocyanin prevention and protection of anti-inflammatory inhibition in Aronia melanocarpa needs further study. It can be deeply studied from the perspective of proteomics and combined with gene knockout technology to accurately find the gene or protein site regulated by anthocyanin.

The digestion, absorption and metabolism of anthocyanins in mice or human body need to be studied. Its metabolic transformation is studied by measuring metabolites in blood and urine.

This paper studies the inhibitory effect of anthocyanin extract of nigrum on inflammation by RT-PCR method $\beta$, tumor necrosis factor- $\alpha$, iNOS, COX2, and reports that the above inflammatory mediators are regulated by $\mathrm{NF}-\kappa \mathrm{B}$. We need to continue to study the next step.

Funding. This project was the key planned project of year 2020 of Department of Science \& Technology of Liaoning province (Serial number2020JH2/10200039).

Conflict of interests. The authors declare that there is no conflict of interests.

\section{REFERENCES}

[1] Ahn, S., Siddiqi, M. H., Noh, H. Y., Kim, Y. J., Kim, Y. J., Jin, C. G., Yang, D. C. (2015): Anti-inflammatory activity of ginsenosides in LPS-stimulated RAW 264.7 cells. - Science Bulletin 60(8): 773-784.

[2] Appel, K., Meiser, P., Millán, E., Collado, J. A., Rose, T., Gras, C. C., Muñoz, E. (2015): Chokeberry (Aronia melanocarpa (Michx.) Elliot) concentrate inhibits NF- $\kappa B$ and synergizes with selenium to inhibit the release of pro-inflammatory mediators in macrophages. - Fitoterapia 105: 73-82.

[3] Bak, M. J., Truong, V. L., Kang, H. S., Jun, M., Jeong, W. S. (2013): Anti-inflammatory effect of procyanidins from wild grape (Vitis amurensis) seeds in LPS-induced RAW 264.7 cells. - Oxidative Medicine \& Cellular Longevity 2013(20): 409321. 
[4] Castañeda-Ovando, A., Pacheco-Hernández, M. D. L., Páez-Hernández, M. E., et al. (2009): Chemical studies of anthocyanins: a review. - Food Chemistry 113(4): 859-871.

[5] Chu, B. F., Lin, H. C., Huang, X. W., Huang, H. Y., Wu, C. P., Kao, M. C. (2016): An ethanol extract of Poria cocos inhibits the proliferation of non-small cell lung cancer A549 cells via the mitochondria-mediated caspase activation pathway. - Journal of Functional Foods 23: 614-627.

[6] Ciocoiu, M., Badescu, L., Badescu, M. (2017): Health Status Improved by Aronia Melanocarpa Polyphenolic Extract. - Soto-Hernández, M. et al (eds.) Phenolic Compounds. IntechOpen, London.

[7] Denev, P. N., Kratchanov, C. G., Ciz, M., Lojek, A., Kratch anova, M. G. (2012): Bioavailability and antioxidant activity of black chokeberry (Aronia melanocarpa) polyphenols: in vitro and in vivo evidences and possible mechanisms of action: a review. - Comprehensive Reviews in Food Science \& Food Safety 11(5): 471-489.

[8] Divya, S. P., Wang, X., Pratheeshkumar, P., Son, Y. O., Roy, R. V., Kim, D., Asha, P. (2015): Blackberry extract inhibits UVB-induced oxidative damage and inflammation through MAP kinases and NF- $\kappa$ B signalling pathways in SKH-1 mice skin. - Toxicology \& Applied Pharmacology 284(1): 92-99.

[9] Fang, Y., Li, X., Li, L., et al. (2015): The role of Nrf2 in protection against Pb-induced oxidative stress and apoptosis in SH-SY5Y cells. - Food \& Chemical Toxicology 86: 191-201.

[10] Gasparrini, M., Forbes-Hernandez, T. Y., Giampieri, F., Afrin, S., Alvarez-Suarez, J. M., Mazzoni, L., Battino, M. (2017): Anti-inflammatory effect of strawberry extract against LPS-induced stress in RAW 264.7 macrophages. - Food \& Chemical Toxicology 102: 110 .

[11] Goh, A. R., Youn, G. S., Yoo, K. Y., Won, M. H., Han, S. Z., Lim, S. S., Park, J. (2016): Aronia melanocarpa concentrate ameliorates pro-inflammatory responses in $\mathrm{HaCaT}$ keratinocytes and 12-O-tetradecanoylphorbol-13-acetate-induced ear edema in mice. Journal of Medicinal Food 19(7): 654.

[12] Hwang, S. J., Kim, Y. W., Park, Y., Lee, H. J., Kim, K. W. (2014a): Anti-inflammatory effects of chlorogenic acid in lipopolysaccharide-stimulated RAW 264.7 cells. Inflammation Research 63(1): 81-90.

[13] Hwang, S. J., Yoon, W. B., Lee, O. H., Cha, S. J., Kim, J. D. (2014b): Radicalscavenging-linked antioxidant activities of extracts from black chokeberry and blueberry cultivated in Korea. - Food Chemistry 146(1): 71.

[14] Isaak, C. K., Petkau, J. C., Blewett, H., K, O., Siow, Y. L. (2017): Lingonberry anthocyanins protect cardiac cells from oxidative-stress-induced apoptosis. - Can J Physiol Pharmacol 95(8): 904-910.

[15] Jakobek, L., Drenjančević, M., Jukić, V., Šeruga, M. (2012): Phenolic acids, flavonols, anthocyanins and antiradical activity of "Nero", "Viking", "Galicianka" and wild chokeberries. - Scientia Horticulturae 147: 56-63.

[16] Jurikova, T., Mlcek, J., Skrovankova, S., Sumczynski, D., Sochor, J., Hlavacova, I., Orsavova, J. (2017): Fruits of black chokeberry Aronia melanocarpa in the prevention of chronic diseases. - Molecules 22(6): 944.

[17] Khan, M. S., Ali, T., Kim, M. W., Jo, M. H., Jo, M. G., Badshah, H., Kim, M. O. (2016): Anthocyanins protect against LPS-induced oxidative stress-mediated neuroinflammation and neurodegeneration in the adult mouse cortex. - Neurochemistry International 100: 110 .

[18] Lee, S. Y., Kim, H. J., Han, J. S. (2013): Anti-inflammatory effect of oyster shell extract in LPS-stimulated raw 264.7 cells. - Preventive Nutrition \& Food Science 18(1): 23.

[19] Lee, H. Y., Jin, B. W., Ryu, G., Yang, W. S., Kim, N. Y., Kim, M. K., Ma, C. J. (2017): Neuroprotective effect of Aronia melanocarpaextract against glutamate-induced oxidative stress in HT22 cells. - Bmc Complementary \& Alternative Medicine 17(1): 207. 
[20] Li, C., Wang, M. H. (2011): Anti-inflammatory effect of the water fraction from hawthorn fruit on LPS-stimulated RAW 264.7 cells. - Nutrition Research \& Practice 5(2): 101.

[21] Liu, Z. G. (2005): Molecular mechanism of TNF signaling and beyond. - Cell Research (English edition) 15(1): 24.

[22] Mcdougall, G. J., Austin, C., Van, S. E., Martin, P. (2016): Salal (Gaultheria shallon) and aronia (Aronia melanocarpa) fruits from Orkney: phenolic content, composition and effect of wine-making. - Food Chemistry 205: 239.

[23] Meng, L. S., Li, B., Li, D. N., et al. (2017): Cyanidin-3-O-glucoside attenuates amyloidbeta (1-40)-induced oxidative stress and apoptosis in SH-SY5Y cells through a Nrf2 mechanism. - Journal of Functional Foods 38: 474-485.

[24] Parzonko, A., Oświt, A., Bazylko, A., Naruszewicz, M. (2015): Anthocyans-rich Aronia melanocarpa extract possesses ability to protect endothelial progenitor cells against angiotensin II induced dysfunction. - Phytomedicine International Journal of Phytotherapy \& Phytopharmacology 22(14): 1238-1246.

[25] Pedro, A. C., Granato, D., Rosso, N. D. (2015): Extraction of anthocyanins and polyphenols from black rice (Oryza sativa L.) by modeling and assessing their reversibility and stability. - Food Chemistry 191: 12-20.

[26] Pellati, F., Benvenuti, S., Magro, L., Melegari, M., Soragni, F. (2004): Analysis of phenolic compounds and radical scavenging activity of echinacea spp. - Journal of Pharmaceutical \& Biomedical Analysis 35(2): 289-301.

[27] Polat, M., Guclu, S. F., Okatan, V., Ercisli, S., Ozaydin, A. G., Colak, A. M., Askin, M. A. (2017): Determination of phenolic compounds in Aronia melanocarpa genotypes grown in Turkey. - Oxidation Communications 40(1): 131-137.

[28] Rop, O., Mlcek, J., Jurikova, T., Valsikova, M., Sochor, J., Reznicek, V., Kramarova, D. (2010): Phenolic content, antioxidant capacity, radical oxygen species scavenging and lipid peroxidation inhibiting activities of extracts of five black chokeberry (Aronia melanocarpa (Michx.) Elliot). - Cultivars 4(22): 2431-2437.

[29] Simić, V. M., Rajković, K. M., Stojičević, S. S., et al. (2016): Optimization of microwave-assisted extraction of total polyphenolic compounds from chokeberries by response surface methodology and artificial neural network. - Separation \& Purification Technology 160: 89-97.

[30] Sivasinprasasn, S., Pantan, R., Thummayot, S., et al. (2016): Cyanidin-3-glucoside attenuates angiotensin II-induced oxidative stress and inflammation in vascular endothelial cells. - Chemico-Biological Interactions 260: 67-74.

[31] Tao, Y., Wang, Y., Pan, M., et al. (2017): Combined ANFIS and numerical methods to simulate ultrasound-assisted extraction of phenolics from chokeberry cultivated in China and analysis of phenolic composition. - Separation \& Purification Technology 178: 178188.

[32] Wang, Y., Li, B., Ma, Y., Wang, X., Zhang, X., Zhang, Q., Meng, X. (2016a): Lonicera caerulea berry extract attenuates lipopolysaccharide induced inflammation in BRL-3A cells: oxidative stress, energy metabolism, hepatic function. - Journal of Functional Foods 24: 1-10.

[33] Wang, Y., Zhu, J., Meng, X., Liu, S., Mu, J., Ning, C. (2016b): Comparison of polyphenol, anthocyanin and antioxidant capacity in four varieties of Lonicera caerulea berry extracts. - Food Chemistry 197(Pt A): 522-529.

[34] Wang, H., Guo, X., Liu, J., Li, T., Fu, X., Liu, R. H. (2017): Comparative suppression of NLRP3 inflammasome activation with LPS-induced inflammation by blueberry extracts (Vaccinium spp.). - Rsc Advances 7(46): 28931-28939.

[35] Xu, W., Zhou, Q., Yao, Y., Li, X., Zhang, J., Su, G., Deng, A. (2016): Inhibitory effect of Gardenblue blueberry (Vaccinium ashei Reade) anthocyanin extracts on lipopolysaccharide-stimulated inflammatory response in RAW 264.7 cells. - J Zhejiang Univ Sci B17(6): 425-436. 
[36] Yaqoob, P., Calder, P. C., Gibney, M. J., Macdonald, I. A., Roche, H. M. (2010): The immune and inflammatory systems. - Nutrition \& Metabolism: 312-338.

[37] Yoon, W. J., Ham, Y. M., Kim, K. N., Park, S. Y., Lee, W. J. (2009): Anti-inflammatory activity of brown alga Dictyota dichotoma in murine macrophage raw 264.7 cells. Journal of Medicinal Plant Research 3(1): 1-8.

[38] Yoon, W. J., Heo, S. J., Han, S. C., Lee, H. J., Kang, G. J., Kang, H. K., Yoo, E. S. (2012): Anti-inflammatory effect of sargachromanol G isolated from Sargassum siliquastrum in RAW 264.7 cells. - Archives of Pharmacal Research 35(8): 1421-1430.

[39] Zdařilová, A., Svobodová, A. R., Chytilová, K., Šimánek, V., Ulrichová, J. (2010): Polyphenolic fraction of Lonicera caerulea L. fruits reduces oxidative stress and inflammatory markers induced by lipopolysaccharide in gingival fibroblasts. - Food \& Chemical Toxicology an International Journal Published for the British Industrial Biological Research Association 48(6): 1555-1561. 